\title{
Are Radical Compartmental Resections for Retroperitoneal Sarcomas Justified?
}

\author{
Chandrajit P. Raut, MD, MSc ${ }^{1}$ and Carol J. Swallow, MD ${ }^{2}$ \\ ${ }^{1}$ Department of Surgery, Brigham and Women's Hospital, Dana-Farber Cancer Institute, and Harvard Medical School, \\ Boston, MA; ${ }^{2}$ Department of Surgery, Mount Sinai Hospital, University of Toronto, Toronto, ON, Canada
}

In this issue, Bonvalot et al. and Gronchi et al. present a follow-up to their recent publications regarding frontline aggressive surgical approach to the management of retroperitoneal sarcomas (RPS). ${ }^{1,2}$ This article addresses the editorial that accompanied the previous two articles, which challenged the authors to show that the mortality and morbidity associated with frontline aggressive surgical approach were modest enough to support the application of this approach in other centers. ${ }^{3}$ This editorial is a direct response to that challenge, with its main stated purpose being to document the complications of frontline aggressive surgical approach.

The authors restrict their analysis to a select group of patients treated in a more recent time period at their own institutions, the Istituto Nazionale Tumori in Milan and the Institute Gustave Roussy, Villejuif near Paris and serving a large metropolitan area. Thus, the authors appropriately target a group of patients who all underwent frontline aggressive surgical approach, although they were not all included in the two previous Journal of Clinical Oncology reports. However, it seems that the same basic methodology that was applied in the original articles was again used in gathering data on operative complications-that is, retrospective review of patient charts. This methodology is notoriously flawed and has been reported to consistently underestimate morbidity by $30-50 \%{ }^{4,5}$ Biases include incompleteness and unreliability of inpatient notes and lack of rigorous follow-up after discharge from the acute-care setting. The authors have partially addressed this by reporting complications that required one or more invasive therapeutic procedures, but they have not included lifethreatening complications that lengthened hospital stay yet

(C) Society of Surgical Oncology 2010

Published Online: 17 April 2010

C. P. Raut, MD, MSc

e-mail: craut@partners.org did not require an invasive procedure (aspiration pneumonia would be one example). Furthermore, each of the authors' institutions serves a large catchment area, and although all patients were apparently seen at the Istituto Nazionale Tumori or the Institute Gustave Roussy at 1 month after discharge, complications in patients who visited other, smaller local centers after discharge may not have been reliably captured. Thus, although we can accept the early postoperative mortality (3\%) and reoperative rates reported here by Bonvalot et al. as accurate, the morbidity rate that they report (18\%) almost certainly greatly underestimates the true rate of serious postoperative complications.

Is the morbidity rate experienced with frontline aggressive surgical approach unexpectedly or unreasonably high? The reoperative rate (12\%) seems high in the view of experts in the field of RPS resection, but that could reflect recall bias and/or differences in local practice with respect to management of leaks, bleeds, and collections. Arguably, we do not have an accurate estimate of what the true complication or reoperation rates are in centers of sarcoma expertise that commonly perform RPS resection but without a routine frontline aggressive surgical approach. Very few prospective trials or series have been reported in RPS, and fewer still have focused attention on postoperative mortality and morbidity. Estimates from the latter include 2-4\% mortality and 21-37\% morbidity. ${ }^{6-9}$ Another complicating factor is that these other series included patients with recurrent RPS, and as any experienced RPS surgeon can attest, resection of recurrent disease is fraught with the specter of life-threatening complications. It is to be remembered that the cases reported here by Bonvalot et al. are all primary RPS, and by inference should be associated with lower operative complication rates. Taken together, these sobering facts heighten the burden of proof for frontline aggressive surgical approach to yield superior long-term oncologic outcomes. 
Another important question is the long-term functional morbidity associated with frontline aggressive surgical approach. In the case of extremity sarcoma, expert centers have published on functional outcomes and quality of life associated with a limb-sparing strategy that makes liberal use of adjuvant radiotherapy. ${ }^{10}$ As with many other tumor types and treatments, clinicians have tended to underestimate the negative impact of cancer therapy on long-term functional capacity and quality of life. In the case of RPS, little to no attention has been turned to this issue. Instances of long-term neuropathy have been reported in association with intraoperative radiotherapy for RPS, but other types of chronic pain, compromise of renal or gastrointestinal function, and musculoskeletal disability have not been measured in any systematic fashion. ${ }^{11,12}$ Composite resection of retroperitoneal muscles, tendons, and nerves as part of frontline aggressive surgical approach procedure would be expected to result in varying degrees of longterm disability. A subset of patients undergoing en bloc nephrectomy would be expected to subsequently develop chronic renal insufficiency, which in turn would alter subsequent chemotherapy treatment options. These too should be important considerations as we weigh the evidence in favor of frontline aggressive surgical approach.

In the present report, the authors also include an analysis of long-term oncologic outcomes in a select patient group, those operated on for primary RPS in a recent era. In keeping with a modest expansion of the frontline aggressive surgical approach groups already reported in their individual Journal of Clinical Oncology publications, the oncologic outcomes reported are essentially the same. Overall survival (OS) at 5 years is $65 \%$, with a crude local recurrence rate of $22 \%$. These results seem relatively favorable compared to many previous series, but the authors' assertion of superiority must be taken with some caution for several reasons. First, it is necessary to compare their results to those reported for primary RPS because primary versus recurrent disease is a recognized prognostic indicator. ${ }^{13}$ Second, one third of the patients included in this series had low-grade tumors, meaning that their cancer-specific survival at 5 years should be close to $90 \% .^{14-16}$ Third, the "long-term" outcomes presented here by Bonvalot et al. are in fact relatively immature because the median follow-up time is only 37 months, with a range of 16-61 months. It is clear that soft tissue sarcoma in the retroperitoneum, unlike that arising at other sites, continues to recur at a steady rate over a 20 -year span after resection. ${ }^{17-19}$ Outcomes at 5 years would have to be markedly different from those reported previously to be considered of potential significance. The combined experience in two prospective trials of neoadjuvant therapy and resection at the M. D. Anderson Cancer Center and Princess Margaret Hospital showed a 5-year OS rate of $61 \%$ in patients with intermediate- and high-grade sarcoma. ${ }^{20}$ With a much longer follow-up interval (median, 89 months), Lehnert and colleagues reported a 5-year local recurrence-free survival of $59 \% .^{21}$ Thus, with the comparatively short follow-up in the present report, we cannot conclude that any perceived difference in OS or local recurrence resulting from the frontline aggressive surgical approach is either statistically or clinically significant. Lack of benefit in OS was the principal reason that amputations for extremity sarcomas have been largely abandoned in favor of limband function-sparing resections coupled with radiotherapy, despite a statistically insignificant trend toward improved local control with the more radical operation. ${ }^{22}$

Can more aggressive or radical resection demonstrably affect oncologic outcomes in RPS? Undoubtedly, the management of RPS is plagued by inadequate resections, particularly in the hands of those who rarely perform such operations, and achievement of total gross resection is a well-acknowledged prognostic factor. ${ }^{16}$ But does frontline aggressive surgical approach actually achieve a wider surgical margin than the typical macroscopically complete resection (resecting involved organs) performed at experienced centers by experienced sarcoma surgeons? Furthermore, even if the margins are cleared more successfully by frontline aggressive surgical approach, does an improvement in OS necessarily ensue? In the present report by Bonvalot and colleagues, frontline aggressive surgical approach usually meant resection of the ipsilateral colon, kidney, and psoas. What constitutes an adequate surgical margin has been the subject of ongoing debate, not only in sarcoma but in other malignancies as well. ${ }^{23,24}$ Achieving negative microscopic margins for RPS resections is challenging. To our knowledge, there are no good guidelines outlining a standardized and reproducible method for the evaluation of margins for RPS. A key problem is that these tumors tend to be quite large at initial presentation, with a median size of $15 \mathrm{~cm}^{25}$

If we think of the retroperitoneum in the most simplistic terms, we can define its three-dimensional margins. The anterior margin is generally the ipsilateral colon and mesocolon, pancreas, liver, or stomach. The posterior margin is generally the psoas and iliacus muscles inferiorly, the ipsilateral kidney and diaphragm superiorly, and the ipsilateral ureter and gonadal vessels medially. However, this is not consistent, and some or all of these structures could be anterior to the mass, in which case they would constitute a portion of the anterior margin. The medial margin usually includes the spine and paraspinous muscles, the inferior vena cava (for right-sided tumors), and the aorta (for left-sided tumors). The lateral margin is constituted by the lateral or flank musculoskeletal sidewall, although depending on the size and location of the tumor, the kidney and/or colon could also border the lateral 
portion of the mass. The superior margin is similarly dependent on the size and location of the mass and may include the diaphragm on either side, the right lobe of the liver, the duodenum, and the uncinate process of the pancreas for right-sided tumors, and pancreatic tail, spleen, and splenic vessels for left-sided tumors. The inferior margin may include the iliopsoas muscle, the femoral nerve, the common, internal, and external iliac vessels, and the pelvic sidewall. Clearly, the size and specific location of the mass determine which of the many structures mentioned above constitute which specific margin.

There is no reason to think that any one of these structures is more critical in determining locoregional failure than any other. In general, the ipsilateral colon and mesocolon and at least a portion of the psoas can be safely and relatively easily resected without much consequence (depending on the patient's prior surgical, radiotherapy, or chemotherapy history). Resection of the kidney, pancreatic tail, and spleen can usually be performed with relatively low short-term morbidity, as the authors show here. Resection of other structures, including but not limited to the aorta, inferior vena cava, iliac vessels, femoral nerve, diaphragm, duodenum, uncinate process, and liver, entail more extensive resections, in general with ensuing greater morbidity. And as one might intuitively predict, Bonvalot et al. found a trend toward increased morbidity with resection that incorporated the head of pancreas, duodenum, or total gastrectomy. Clearance of margins along great vessels is perhaps most problematic of all of the challenges in RPS surgery, and resection of these structures indeed predicted a higher complication rate. It is for this reason, among several others, that many consider adjuvant or neoadjuvant radiotherapy as a potential treatment for improving locoregional control in RPS.

Determining how thoroughly a surgeon has cleared any of these margins requires a meticulous evaluation by the pathologist, beginning with the unfixed specimen. Previous studies have discussed the inadequate assessment of circumferential margins in pancreatic cancer. ${ }^{26,27}$ Studies reporting the results of pancreaticoduodenectomies generally mention whether margins are positive or negative but do not commonly describe which specific margins are positive and which are negative. The American Joint Committee on Cancer staging manual details how pancreaticoduodenectomy specimen margins should be assessed, and several studies have described a detailed approach to this. ${ }^{26,28,29}$ Nevertheless, only rarely do articles that report oncologic outcomes clearly discuss such details. In RPS, no guidelines for the assessment of margins of resection have been developed as of yet, although some centers do report their microscopic margin positivity rates. However, many clinicians in the field question the accuracy of microscopic margin analysis in tumors of the size and relations typical of RPS.

Can margin status be viewed as a surrogate for longterm oncologic outcomes in RPS? Several major groups have reported that margin status predicts survival, but for the most part, it is R2 status that determines this marked difference, whereas the effect of R1 status is negligible or nonexistent. ${ }^{15}$ One can legitimately question the benefit of achieving a wide microscopic margin along two or three surfaces by removing additional easily disposable organs, while performing what is essentially a marginal excision along another surface that approximates critical structures (for instance, superior mesenteric artery, duodenum, head of pancreas, or femoral nerve). One way to settle the question of whether microscopic margins matter would be to compare the results of RPS resection (frontline aggressive surgical approach versus standard-of-care macroscopically complete resection) performed by experienced sarcoma surgeons and evaluated by experienced sarcoma pathologists with a detailed description of which margins are positive and negative, a correlation between pattern of treatment failure and margin positivity, and short- and long-term complications. However, such a study would be challenging if not impossible to conduct.

Ultimately, if the burden of marginal viable tumor cells left behind in the field can be reduced by less harmful means, surely that constitutes a strategy worth pursuing. It is here that preoperative radiotherapy may offer benefit, although this has yet to be demonstrated in a randomized prospective trial. Radiotherapy delivered to the margins of concern, either before or after surgery, may trump any radical extent of surgery. Sadly, the American College of Surgeons Oncology Group trial that addressed this salient question was closed prematurely as a result of poor accrual. Perhaps a renewed global effort to answer this question, learning from previous trial attempts, may be most beneficial. We support efforts by the European oncology community to launch such a trial, perhaps to be carried out in high-volume centers that already have experience with preoperative radiation. Bonvalot et al. are ideally placed to lead such an effort.

\section{REFERENCES}

1. Bonvalot S, Rivoire M, Castaing M, et al. Primary retroperitoneal sarcomas: a multivariate analysis of surgical factors associated with local control. J Clin Oncol. 2009;27:31-7.

2. Gronchi A, Lo Vullo S, Fiore M, et al. Aggressive surgical policies in a retrospectively reviewed single-institution case series of retroperitoneal soft tissue sarcoma patients. J Clin Oncol. 2009;27:24-30.

3. Pisters PW. Resection of some-but not all-clinically uninvolved adjacent viscera as part of surgery for retroperitoneal soft tissue sarcomas. J Clin Oncol. 2009;27:6-8. 
4. Weigelt JA, Dryer D, Haley RW. The necessity and efficiency of wound surveillance after discharge. Arch Surg. 1992;127:77-81.

5. Kent P, McDonald M, Harris O, Mason T, Spelman D. Postdischarge surgical wound infection surveillance in a provincial hospital: follow-up rates, validity of data and review of the literature. ANZ J Surg. 2001;71:583-9.

6. Jones JJ, Catton CN, O'Sullivan B, et al. Initial results of a trial of preoperative external-beam radiation therapy and postoperative brachytherapy for retroperitoneal sarcoma. Ann Surg Oncol. 2002;9:346-54.

7. Lewis JJ, Leung D, Woodruff JM, Brennan MF. Retroperitoneal soft-tissue sarcoma: analysis of 500 patients treated and followed at a single institution. Ann Surg. 1998;228:355-65.

8. Bussieres E, Stockle EP, Richaud PM, et al. Retroperitoneal soft tissue sarcomas: a pilot study of intraoperative radiation therapy. J Surg Oncol. 1996;62:49-56.

9. Dziewirski W, Rutkowski P, Nowecki ZI, et al. Surgery combined with intraoperative brachytherapy in the treatment of retroperitoneal sarcomas. Ann Surg Oncol. 2006;13:245-52.

10. Davis AM, O'Sullivan B, Turcotte R, et al. Late radiation morbidity following randomization to preoperative versus postoperative radiotherapy in extremity soft tissue sarcoma. Radiother Oncol. 2005;75:48-53.

11. Krempien R, Roeder F, Oertel S, et al. Intraoperative electronbeam therapy for primary and recurrent retroperitoneal soft-tissue sarcoma. Int J Radiat Oncol Biol Phys. 2006;65:773-9.

12. Petersen IA, Haddock MG, Donohue JH, et al. Use of intraoperative electron beam radiotherapy in the management of retroperitoneal soft tissue sarcomas. Int J Radiat Oncol Biol Phys. 2002;52:469-75.

13. Anaya DA, Lahat G, Liu J, et al. Multifocality in retroperitoneal sarcoma: a prognostic factor critical to surgical decision-making. Ann Surg. 2009;249:137-42.

14. Ballo MT, Zagars GK, Pollock RE, et al. Retroperitoneal soft tissue sarcoma: an analysis of radiation and surgical treatment. Int J Radiat Oncol Biol Phys. 2007;67:158-63.

15. Singer S, Antonescu CR, Riedel E, Brennan MF. Histologic subtype and margin of resection predict pattern of recurrence and survival for retroperitoneal liposarcoma. Ann Surg. 2003;238:358-70.

16. van Dalen T, Hennipman A, Van Coevorden F, et al. Evaluation of a clinically applicable post-surgical classification system for primary retroperitoneal soft-tissue sarcoma. Ann Surg Oncol. 2004;11:483-90.
17. Catton CN, O'Sullivan B, Kotwall C, et al. Outcome and prognosis in retroperitoneal soft tissue sarcoma. Int J Radiat Oncol Biol Phys. 1994;29:1005-10.

18. Dalal KM, Kattan MW, Antonescu CR, Brennan MF, Singer S. Subtype specific prognostic nomogram for patients with primary liposarcoma of the retroperitoneum, extremity, or trunk. Ann Surg. 2006;244:381-91.

19. Heslin MJ, Lewis JJ, Nadler E, et al. Prognostic factors associated with long-term survival for retroperitoneal sarcoma: implications for management. J Clin Oncol. 1997;15:2832-9.

20. Pawlik TM, Pisters PW, Mikula L, et al. Long-term results of two prospective trials of preoperative external beam radiotherapy for localized intermediate- or high-grade retroperitoneal soft tissue sarcoma. Ann Surg Oncol. 2006;13:508-17.

21. Lehnert T, Cardona S, Hinz U, et al. Primary and locally recurrent retroperitoneal soft-tissue sarcoma: local control and survival. Eur J Surg Oncol. 2009;35:986-93.

22. Rosenberg SA, Tepper J, Glatstein E, et al. The treatment of softtissue sarcomas of the extremities: prospective randomized evaluations of (1) limb-sparing surgery plus radiation therapy compared with amputation and (2) the role of adjuvant chemotherapy. Ann Surg. 1982;196:305-15.

23. Gerrand $\mathrm{CH}$, Wunder JS, Kandel RA, et al. Classification of positive margins after resection of soft-tissue sarcoma of the limb predicts the risk of local recurrence. J Bone Joint Surg Br. 2001;83:1149-55.

24. Sampo M, Tarkkanen M, Huuhtanen R, et al. Impact of the smallest surgical margin on local control in soft tissue sarcoma. Br J Surg. 2008;95:237-43.

25. Stoeckle E, Coindre JM, Bonvalot S, et al. Prognostic factors in retroperitoneal sarcoma: a multivariate analysis of a series of 165 patients of the French Cancer Center Federation Sarcoma Group. Cancer. 2001;92:359-68.

26. Raut CP, Tseng JF, Sun CC, et al. Impact of resection status on pattern of failure and survival after pancreaticoduodenectomy for pancreatic adenocarcinoma. Ann Surg. 2007;246:52-60.

27. Staley CA, Cleary KR, Abbruzzese JL, et al. The need for standardized pathologic staging of pancreaticoduodenectomy specimens. Pancreas. 1996;12:373-80.

28. Chang DK, Johns AL, Merrett ND, et al. Margin clearance and outcome in resected pancreatic cancer. J Clin Oncol. 2009;27:2855-62.

29. Verbeke CS, Leitch D, Menon KV, et al. Redefining the R1 resection in pancreatic cancer. Br J Surg. 2006;93:1232-7. 